TP Periodica Polytechnica Electrical Engineering and Computer Science

59(3), pp. 132-137, 2015

DOI: $10.3311 /$ PPee. 8582

Creative Commons Attribution (1)

RESEARCH ARTICLE

\section{Efficient Formulation of Minimum Income Condition Orders on the All-European Power Exchange}

\author{
Ádám Sleisz ${ }^{*}$, Dávid Raisz ${ }^{1}$
}

Received 17 May 2015; accepted after revision 21 August 2015

\begin{abstract}
The unification of European day-ahead electricity markets leads to several challenging problems some of which arise from the mathematical formulation of the common power exchange $(P X)$. The officially proposed clearing algorithm - called EUPHEMIA - is a hybrid framework containing diverse and quite complicated elements. The straightforward formulation of a few bid types - most importantly, the Minimum Income Condition (MIC) orders for suppliers - would refer the clearing into the category of non-convex mixed integer quadratically constrained programs (MIQCPS) which is one of the hardest class of optimization problems.

Several attempts have been made to efficiently execute the clearing but the question is not yet finally answered. A new formulation of MIC orders is presented to overcome the main hurdles making MIC orders a relatively simple addition to the usual convex mixed integer quadratic programming (MIQP) model of European PXs. Theoretical advances are discussed and initial tests are also provided for demonstration.
\end{abstract}

\section{Keywords}

power exchange, economic modeling, mixed integer optimization, power plant, Internal Energy Market

\section{Introduction}

Although the apparent aim of the European power exchange (PX) unification process is the more flexible exploitation of the grid in international electricity trade, the inclusion of the most advanced and innovative market concepts is also attempted [1]. Among these, several new order types are introduced, mostly to be used by power suppliers to take some of their binding technical and economic characteristics into account.

Complicated properties of power generation has been one of the most challenging issues in electricity market design since the appearance of the subject [2]. The expenses of building, operating, maintaining and decommissioning power plants are ideally handled by market forces meaning that various fixed and variable costs are associated with every unit of supplied energy. On the other hand, several technical constraints are also present which can make daily trading rather difficult e.g. minimal operating loads and load gradient limitations.

The main hindrance arising from these considerations is that the overall economic model of power plants is generally nonconvex in nature. The clearing of PXs is usually formalized as optimization problems, and non-convex elements make these problems substantially harder to solve. Thus, the introduction of non-convexities is unavoidable to provide feasible clearing results for suppliers but the computational burden evoked by them must be reduced as much as possible. This trade-off can be assessed by careful formulation of supply order types such as different kinds of block orders.

Complex orders can offer an alternative approach to block orders. They originate from the Iberian PX and can be considered a novel concept in the mainstream European setting. They have their potentially non-convex element - the so-called Minimum Income Condition (MIC) - and can provide a sensible improvement in the economic model of power supply compared to block orders. However, the efficient implementation of MICs is not yet fully resolved.

Section 2 provides the definition of complex orders with MICs (also called MIC orders), and the consequences of their straightforward formulation. Previous attempts to handle this kind of offers are evaluated in Section 3 while a new, practically superior
${ }^{1}$ Department of Electric Power Engineering,

Budapest University of Technology and Economics

H-1111 Budapest, Egry József str. 18., Hungary

*Corresponding author, e-mail: sleisz.adam@vet.bme.hu 
method is described in Section 4. Demonstrative test results and conclusions are presented in Sections 5 and 6, respectively.

\section{The issue of Minimum Income Conditions}

Complex orders are supply orders consisting of several hourly step bids for potentially different market hours. These can be bound together by additional conditions e.g. MICs, Load Gradient Conditions (LGCs) and Scheduled Stop Conditions (SSCs). This paper considers the case when LGCs are not associated with the complex bid set.

The set of individual hourly bids consists of two subsets: the MIC bid set $(S M)$ and the SSC bid set (SS). The MIC prescribes that the overall income of the MIC order must cover its given costs if any quantity of the bids in $S M$ is accepted (1).

$$
\sum_{i \in S M} A M_{i}>0 \Rightarrow F T+V T \cdot Q A \leq I N C O M E
$$

In this formula, $A M_{i}$ denotes the non-negative acceptance values of individual orders in the MIC bid set while FT and VT are the fix and variable cost parameters defined by the bidder. $Q A$ and $I N C O M E$ are auxiliary variables corresponding to the total allocated volume of the MIC order and its total income.

$$
\begin{gathered}
Q A=\sum_{i \in S M} A M_{i} q_{i}+\sum_{i \in S S} A S_{i} q_{i} \\
I N C O M E=\sum_{i \in S M} I N M_{i}+\sum_{i \in S S} I N S_{i}
\end{gathered}
$$

As $A M_{i}$ and $I N M_{i}$ are the acceptance and collected income values of the individual orders in the MIC set, so denote $A S_{i}$ and $I N S_{i}$ the same for the bids in the SSC set. $q_{k}$ indicates the offered quantity in the corresponding elementary bid. The above equations show that SSC actually relaxes the criterion of the MIC. The bids in SS are inserted into the income calculation but they are not necessarily rejected if costs are too large to be covered by incomes. This kind of operation can be advantageous in the first hours of the complex order because it can provide a smooth stop schedule in the case of MIC violation.

For every hour $h, I N M$ s and INSs can be expressed as:

$$
\begin{gathered}
\forall k \in S M_{h}: I N M_{k}=A M_{k} q_{k} M C P_{h} \\
\forall k \in S S_{h}: I N S_{k}=A S_{k} q_{k} M C P_{h}
\end{gathered}
$$

$S M_{h}$ and $S S_{h}$ represent the hourly decomposition of $S M$ and $S S$ bid sets, respectively, while $M C P_{h}$ is the corresponding market clearing price in hour $h$.

\subsection{Pricing of individual bids}

The pricing of orders in the MIC set is the same as the pricing of any hourly order if the MIC is satisfied [1]. Otherwise, they are all rejected.

$$
\begin{aligned}
& \forall k \in S M_{h}: \sum_{i \in S M} A M_{i}>0 \& A M_{k}>0 \Rightarrow M C P_{h} \geq p_{k} \\
& \forall k \in S M_{h}: \sum_{i \in S M} A M_{i}>0 \& A M_{k}<1 \Rightarrow M C P_{h} \leq p_{k}
\end{aligned}
$$

In the above equations, $p_{k}$ is the offered price of the bid in question. Note again that only step orders are allowed into the MIC and SSC sets [1].

Offers in the SSC set are ordinary hourly step orders:

$$
\begin{aligned}
& \forall k \in S S_{h}: A S_{k}>0 \Rightarrow M C P_{h} \geq p_{k} \\
& \forall k \in S S_{h}: A S_{k}<1 \Rightarrow M C P_{h} \leq p_{k}
\end{aligned}
$$

\subsection{Computational obstacles}

The calculations of individual bid incomes in (4) and (5) contain bilinear expressions of bid acceptance (either $A M_{k}$ or $\left.A S_{k}\right)$ and market price $\left(M C P_{h}\right)$ variables. Bilinear terms are non-convex on any rectangular region of their domain [3]. Moreover, this kind of non-convex elements are fundamentally different from those of earlier non-convex order types in Europe i.e. block orders [4].

The non-convexity of block bids arise from the indivisibility (fill-or-kill) property of their bid volume, therefore it is discrete in nature. Since the continuous relaxation of the problem remains convex, the resulting mathematical program belongs to the category of convex mixed integer quadratic programs (MIQPs) [1]. This means that the number of potential local optima is finite, and the solution search is usually manageable by some version of the branch-and-bound metaheuristic (B\&B).

On the other hand, the decision variables in the bilinear terms of (4) and (5) have continuous domains. Thus, in the presence of MICs, even the continuous relaxation of PX clearing can be non-convex classifying the clearing as a non-convex mixed integer quadratically constrained program (MIQCP). Widely used generic B\&B routines are not capable of solving this kind of problems. Possible solution methods have to heavily rely on problem-specific knowledge but they are still generally more complicated and probably very inefficient [4-5].

\section{Earlier solution attempts}

The official description of EUPHEMIA [1] includes a solution procedure for the arising optimization problem. This procedure is basically a B\&B search in which the MICs of complex orders are handled in a specific way. The constraints playing the role of (1) are removed from the original clearing model, and their satisfaction is inspected only when a new incumbent is found.

The algorithm itself cannot be reproduced from the description because no detailed mechanisms are presented for the case of MIC violation. Nonetheless, it is a problem-specific heuristic method, thus it needs to pass through a particularly profound 
verification process before any practical application and probably lacks several advantages of exact optimization.

\subsection{Primal-dual framework}

The clearing of conventional European PXs is usually carried out using a MIQP model in which acceptance values and market prices are all primal decision variables. This framework dictates that the connection between quantities and prices must be explicitly defined as logical constraints such as (8) or (9). However, other approaches are also available; the same economic function can be performed by substantially different mathematical formulations.

In North America, only quantity variables are present in primal PX clearing equations while price variables arise from the dual problem [6]. The transparency and overall performance of this approach is remarkable but its direct European application is prevented by specific properties of the market environment (most importantly, by the presence of self-scheduling generators). However, a conceptually similar framework is available [7], and it is capable of clearing not only hourly and block bids but also complex orders with associated MICs.

The proposed model incorporates all quantity and price variables in the original primal problem. The main discrepancy from the usual framework is that their connection is not prescribed by logical constraints; it is ensured by duality theory instead. A titular "primal" problem is constructed from quantity equations and while a titular "dual" one is built from the corresponding dual price constraints. All of these conditions are represented on equal footing in the actual primal model.

According to duality theory, optimality is proven if the objectives of primal and dual problems are equal. This condition is rather easy to specify for our titular model parts. MICs imply additional connections between certain "primal" and "dual" variables, and their satisfaction can be prescribed with linear expressions.

This primal-dual framework has its unquestionable advantages. It reduces the number of non-convex elements to the minimum: one integer variable belongs to each block and each complex order while logical constraints are eradicated with all their auxiliary binary indicator variables. The resulting mathematical program is actually a convex mixed integer linear program (MILP) which lies within the usual scope of generic $\mathrm{B} \& \mathrm{~B}$ routines.

On the other hand, serious deficiencies are present as well. Since optimality is ensured by one of the constraints containing the expression of the objective, the expression in question has to be linear. This means that only step orders are available as ordinary hourly orders, an obvious deviation from the original EUPHEMIA specification. The option of SSC is also excluded from the formalized complex orders. One of the other important drawbacks of this formulation may be that it is not compatible with functioning European clearing mechanisms, therefore it would render previously acquired computational experience unusable.

\subsection{Continuous non-convex techniques}

An easily observable fact is that the income of a bid depends on its acceptance. The connection between acceptance rules and income calculations can be exploited to find a feasible solution method for MIC orders [8]. The solution search itself is based on the techniques of convex envelopes, spatial branching and a problem-specific domain reduction strategy [4]. Details of these mathematical tools and procedures are beyond the scope of this paper.

Though the theoretical efficiency of the algorithm of [8] is the same as previously used convex MIQPs, the implementation of problem-specific parts make it questionable in certain market situations. Similarly to the primal-dual approach, the solution search is not simply compatible with conventional European PXs. On the other hand, linear hourly bids and complex orders with the SSC option can be available for participants on the corresponding exchange.

\section{The proposed formulation}

The main thread in [8] can be continued with an even closer examination of possible bid incomes. It can be recognized that an income value is not only connected to the corresponding bid acceptance but the latter can be used to decompose the income calculation into two convex subcases.

In every hour $h$, for every bid $k$ in the hourly MIC set:

$$
\begin{aligned}
& \sum_{i \in S M} A M_{i}>0 \& A M_{k}>0 \Rightarrow I N M_{k}=I N M n r_{k} \\
& \sum_{i \in S M} A M_{i}>0 \& A M_{k}<1 \Rightarrow I N M_{k}=I N M n a_{k}
\end{aligned}
$$

In these formulas, $I N M n r_{k}$ denotes the bid income if the corresponding bid is not fully rejected while INMna $a_{k}$ has the same meaning if the bid is not fully accepted.

$$
\begin{gathered}
I N M n r_{k}=q_{k} \cdot M C P_{h}+A M_{k} \cdot q_{k} \cdot p_{k}-q_{k} \cdot p_{k} \\
I N M n a_{k}=A M_{k} \cdot q_{k} \cdot p_{k}
\end{gathered}
$$

The income expressions can be investigated with three different scenarios in mind:

- If the bid is fully accepted, constraint (10) is active while (11) is not. The calculated income $\left(I N M n r_{k}\right)$ equals the product of the full bid quantity and the corresponding market price because $A M_{k}=1$.

- If the bid is partially accepted, both (10) and (11) are active, thus $I N M n r_{k}$ and INMna $a_{k}$ have to be both equal to the product of the accepted bid quantity and the bid price. INMna $a_{k}$ trivially satisfies this criterion. Partial 
acceptance is possible only if $M C P_{h}=p_{k}$, therefore $I N M n r_{k}$ is correct as well.

- If the bid is fully rejected, constraint (11) is active and (10) is not. The calculated income $\left(I N M n a_{k}\right)$ is zero because $A M_{k}=0$.

All scenarios are handled properly with linear equations. The constraints of the SSC bids can be found very similarly (in every hour $h$, for every bid $k$ in $S S_{h}$ ):

$$
\begin{gathered}
A S_{k}>0 \Rightarrow I N S_{k}=I N S n r_{k} \\
A S_{k}<1 \Rightarrow I N S_{k}=I N S n a_{k} \\
I N S n r_{k}=q_{k} \cdot M C P_{h}+A S_{k} \cdot q_{k} \cdot p_{k}-q_{k} \cdot p_{k} \\
I N S n a_{k}=A S_{k} \cdot q_{k} \cdot p_{k}
\end{gathered}
$$

The proposed mathematical model of MIC orders consists of the linear constraints (1)-(3) and (6)-(17).

\subsection{Theoretic analysis}

The suggested formulation is entirely linear, therefore the usual convex MIQP framework of European PXs can be used without any further modification. Despite the apparent nonconvexity and seemingly complicated nature of the orders in question, they can be composed as a relatively easy and efficient addition.

It has to be noted, however, that the income calculation heavily relies upon the acceptance rules of individual bids i.e. it is not appropriate for general application with other order types. The applicable clearing of complex orders with LGCs remains out of reach as well.

\section{Numerical tests}

Since the European PX unification process is still underway, real (and certainly, public) test data do not exist for development purposes concerning the clearing algorithm. Therefore, the performance of the proposed MIC formulation is demonstrated on an artificially created sample.

The data preparation and manipulation tasks are implemented in MATLAB environment while the actual clearing problems are solved by the academic version of the IBM ILOG CPLEX software package.

\subsection{Test scenario}

The test sample belongs to an imaginary exchange which handles the ATC-based coupling of two countries' separate markets. Average system load of Country $\mathrm{X}$ is around $3 \mathrm{GW}$ which is substantially smaller than the approximately $10 \mathrm{GW}$ average of Country Y. The available capacity of the connection link varies randomly between $500 \mathrm{MW}$ and $1500 \mathrm{MW}$ in both directions.
The structure of power generation is quite different in the two price regions. Country $\mathrm{X}$ has approximately $1000 \mathrm{MW}$ spare generation capacity compared to expected loads, and it also follows a more distributed production model with relatively large number of small units. Small-scale generation is represented with convex hourly bids in this simulation because it is assumed to be generally more flexible, and also because the minor volumes of single suppliers reduce the likability of individually detrimental clearing results.

Seven trading days are considered; the fundamental timedependent variation of submitted orders is based on a one week period of the real power consumption sample also used in [9]. Aggregated bid price curves are constructed from targeted load values with the help of appropriately adjusted and linearized sigmoid functions. The bidding model of large power plants is not subjected to this kind of manipulations, they are considered with constant basic parameters throughout the whole week.

The main structure of supply and demand, and relative importance of different order types are summarized in Table 1. Indicated market share values are estimations because random elements play crucial role during the data preparation process; they are especially important in the case of non-convex order types. The number, the quantity profiles and prices of block bids all have rather wide ranges, however, the relative importance of blocks is clearly reduced by the presence of complex orders. The variance of MIC cost terms (FTs and VTs $)$ is also rather high in order to simulate more clearing scenarios for these orders. The SSC sets contain the cheapest bids of the complex orders' first hours on each day.

Table 1 Main properties of submitted orders in the test scenario

\begin{tabular}{ccccc}
\hline \multirow{2}{*}{ Order type } & $\begin{array}{c}\text { Derived from } \\
\text { load data }\end{array}$ & \multicolumn{2}{c}{ Market share } \\
\cline { 3 - 5 } & hourly & yes & $\sim 47 \%$ & $\sim 29 \%$ \\
Supply & block & no & $\sim 7 \%$ & $\sim 3 \%$ \\
& complex & no & $\sim 46 \%$ & $\sim 68 \%$ \\
\hline \multirow{2}{*}{ Demand } & hourly & yes & $\sim 93 \%$ & $\sim 97 \%$ \\
& block & no & $\sim 7 \%$ & $\sim 3 \%$ \\
\hline
\end{tabular}

The prevalence of hourly supply orders in Country X can be attributed to the pronounced role of small scale generation. Complex orders represent all of the major power plants: there are two of them in Country $\mathrm{X}$ and five in Country Y. Most of these have $1000 \mathrm{MW}$ production capacity, though Country $\mathrm{Y}$ has two $2000 \mathrm{MW}$ stations as well. Price curves of complex bids are quite simple with two or three steps in the usual $30 €$ to $90 €$ interval. 

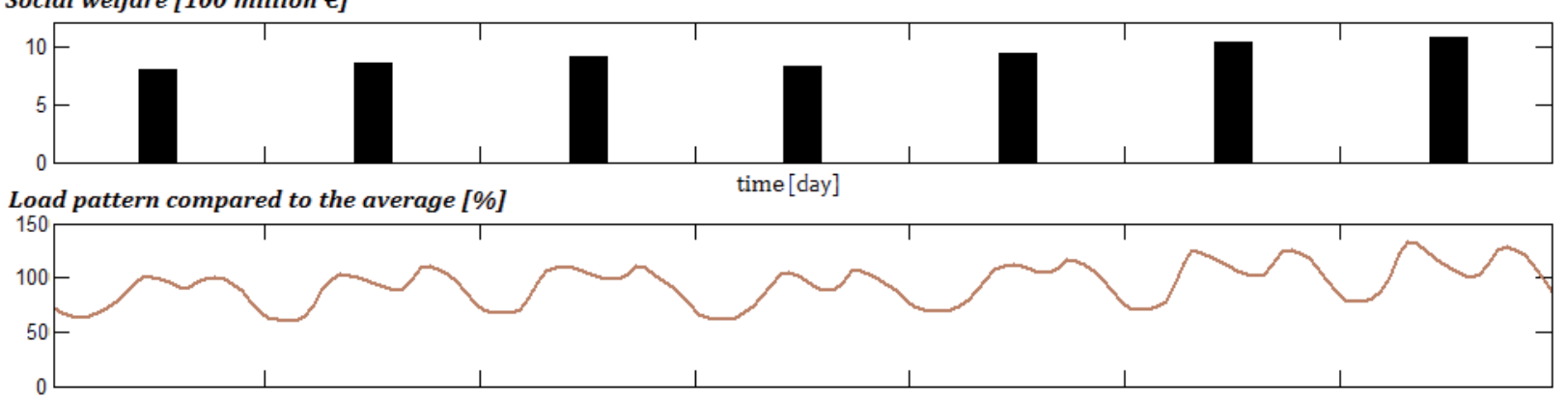

Market clearing prices in Country $X$ and Country $Y$ [€/MWh]

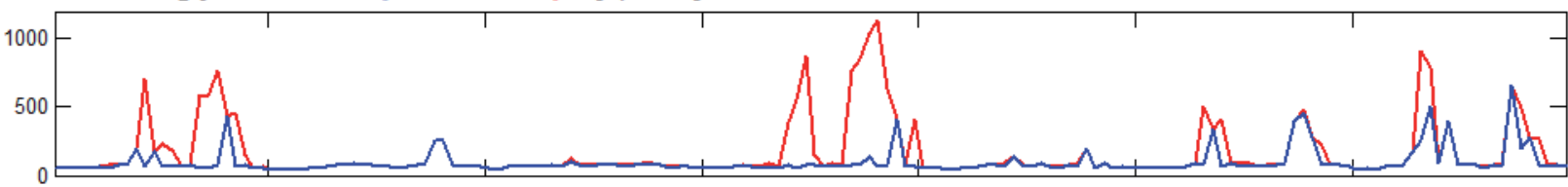

Energy transmission on the connection line and corresponding ATC limits [MWh]

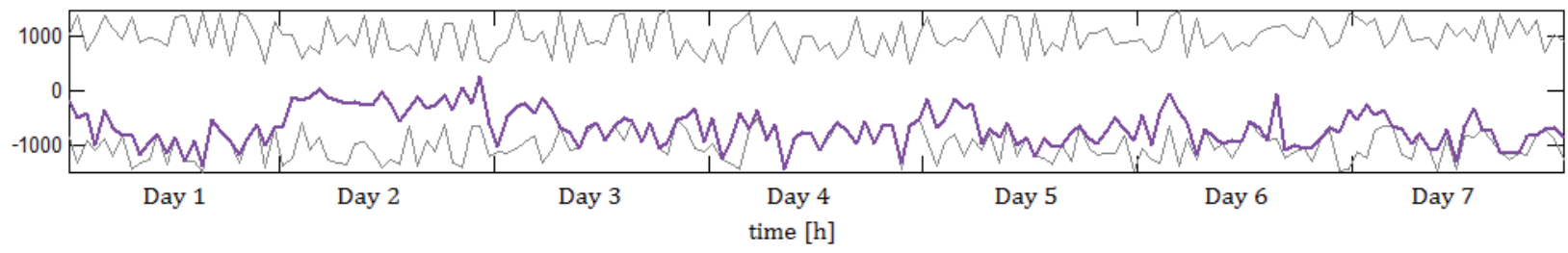

Fig. 1 Illustration of test results.

\subsection{Results and discussion}

The arising clearing problems have been solved by the generic convex MIQP solver of CPLEX. The 10 minute time limit was breached on Day 1, 2, 4 and 5 i.e. these solution processes were terminated before optimality could be proven. However, the B\&B gap was smaller than $1 \%$ in each case meaning that any remaining objective function (social welfare) improvements would not be significant.

Results are illustrated in Fig. 1. Since a substantial part of the supply is provided by orders with basically constant parameters, a relatively strong correlation between daily load and social welfare can be expected. This is observable in Fig. 1. The graphs of $M C P \mathrm{~s}$ and energy transmissions show other notable test results. For example, the energy transmission could be assumed to be mainly negative, because only Country X has generation capacity to share and the nominal direction of the connection line points to Country X from Country Y. This conjecture is also verified by the clearing solutions.

The obtrusive price spikes on certain days are caused by the relatively inelastic supply provided by hourly orders. In the peak hours of Day 6 and 7, system load greatly exceeds the average, therefore the energy from large production units are insufficient to cover the whole heavy demand leaving the remainder for more expensive hourly supply orders to match.

On the other hand, no conspicuous demand spike is present on Day 1 and Day 4. Unsteady prices on these days can be explained by the behaviour of complex orders. One of the 2000 MW complex orders is refused on both days due to its MIC. This decision causes severe energy shortage in Country $Y$ which cannot be fulfilled by the excess production in Country $\mathrm{X}$ through the bottleneck of the connection line.

There is another rejected MIC order in the clearing solution of Day 2 and its effects are also visible in Fig. 1. One of the $1000 \mathrm{MW}$ power stations in Country $\mathrm{X}$ are out of operation i.e. the extra supply is removed from the sys-tem. As a result, this is the only day when the usage of the interconnection is quite low, moreover, some of the hourly energy exchanges occur in the positive direction.

\section{Conclusion}

Complex supply orders with MICs and SSCs are expected to be essential parts of the all-European market design. They provide a quite fitting model for the economic interests of resource providers. A new formulation is presented which can be a significant step to eliminate computational hindrances in the clearing process of these offers. The proposed model is computationally efficient as MIC orders become a simple, linear addition to the usual convex MIQP framework. A basic comparative analysis of other modelling attempts and test demonstrations are also included in the paper.

The logical continuation of this research is to find working formulations for LGCs in complex orders and examine the several alternative concepts described in [10]. The inspection of different bid pricing regimes can be relevant from the viewpoint of future market designs. Meanwhile, the relative advantages and drawbacks of different non-convex order types can also be investigated which can be a rather important topic for trading power plants. 


\section{Acknowledgment}

The authors gratefully acknowledge the contributions of Dániel Divényi, Beáta Polgári and Péter Sörés for their valuable feedback remarks and suggestions concerning the research and the manuscript.

\section{References}

[1] "EUPHEMIA Public Description. PCR Market Coupling Algorithm." [Online] Available from: http:/www.belpex.be/wp-content/uploads/Euphemia-public-description-Nov-2013.pdf 2013. [Accessed: 17th May 2015]

[2] Meeus, L. "Power Exchange Auction Trading Platform Design." Leuven: Katholieke Universiteit. 2006.

[3] McCormick. G. P. "Computability of global solutions to factorable nonconvex programs: Part I - Convex underestimating problems." Mathematical Programming. 10 (1). pp 147-175. 1976. DOI: $10.1007 / \mathrm{bf0} 1580665$

[4] Sleisz, Á., Sorrés, P., Raisz, D. "Algorithmic Properties of the All-European Day-Ahead Electricity Market." In: European Energy Market (EEM), 2014 11th International Conference on the. pp. 1-6, 28-30 May 2014. DOI: 10.1109/EEM.2014.6861275
[5] Burer, S., Letchford, A. N. "Non-convex mixed-integer nonlinear programming: A survey." Surveys in Operations Research and Management Science. 17 (2). pp.97-106.2012. DOI: 10.1016/j.sorms.2012.08.001

[6] Wu, T., Rothleder, M., Alaywan, Z., Papalexopoulos, A. D. "Pricing Energy and Ancillary Services in Integrated Market Systems by an Optimal Power Flow." IEEE Transactions on Power Systems. 19 (1). pp. 339-347. 2004. DOI: 10.1109/tpwrs.2003.820701

[7] Madani, M., Van Vyve, M. "A primal-dual framework for non-convex dayahead electricity auctions with uniform prices." [Online] Available from: http://arxiv.org/pdf/1410.4468v1.pdf 2014. [Accessed: 17th May 2015]

[8] Sleisz, Á., Raisz, D. "Clearing Algorithm of Minimum Income Condition Orders on European Power Exchanges." In: 55th International Scientific Conference on Power and Electrical Engineering of Riga Technical University (RTUCON14). Riga, Latvia, 14 October 2014.

[9] Amjady, N., Keynia, F. "Short-term load forecasting of power systems by combination of wavelet transform and neuro-evolutionary algorithm." Energy. 34 (1). pp. 46-57. 2009. DOI: 10.1016/j.energy.2008.09.020

[10] Sleisz, Á., Divényi, D., Polgári, B., Sőrés, P., Raisz, D. "Challenges in the Formulation of Complex Orders on European Power Exchanges." In: 12th International Conference on the European Energy Market (EEM15). Lisbon. 2015. 